\title{
Combining Ability and Heterosis Analysis of Single Cross Hybrids of Maize (Zea mays L.)
}

\author{
Siddharth Panda ${ }^{1 *}$, Mruthunjaya C. Wali ${ }^{1}$, R.M. Kachapur ${ }^{2}$ and S.I. Harlapur ${ }^{2}$ \\ ${ }^{1}$ Department of Genetics and Plant Breeding, College of Agriculture, UAS, \\ Dharwad-580005, Karnataka, India \\ ${ }^{2}$ All India Coordinated Research Project on Maize, MARS, Dharwad-580005, Karnataka, India \\ *Corresponding author
}

\section{A B S T R A C T}

\begin{tabular}{|c|}
\hline Keywords \\
\hline $\begin{array}{l}\text { Maize, Line X } \\
\text { tester, Pooled } \\
\text { analysis, Diverse } \\
\text { environments. }\end{array}$ \\
\hline Article Info \\
\hline $\begin{array}{l}\text { Accepted: } \\
\text { 23 September } 2017 \\
\text { Available Online: } \\
10 \text { October } 2017\end{array}$ \\
\hline
\end{tabular}

A study was carried out using 48single cross experimental maize hybrids developed from 16 lines (with high test weight and per se performance) and three testers crossed in Line $\times$ Tester design and were evaluated at three diverse environments, viz. Arabhavi (Northern dry zone), Agriculture Research Stations of Nippani and Main Agricultural Research Station (MARS) Dharwad (Northern transition zone) in Karnataka, for their grain yield stability. The hybrids along with 3 checks; GH-0727, CP-818 and NK-6240 were sown in Randomised Block Design across the three locations during kharif-2016. Line $\mathrm{X}$ tester analysis, pooled over locations was run on Windostat, version 8.1 using the different yield and yield attributing traits that were observed during the crop growth and postharvest stages. P-679 proved to be a promising combiner for most of the yield and yield attributing traits. Hybrids; GH-1514 and GH-1532 were superior crosses with highest yield across the three locations.

\section{Introduction}

Maize (Zea mays L.) is a short duration high yielding cereal crop and has key importance in assuring the world food security (Saeed et al., 2000). Being a $\mathrm{C}_{4}$ plant, it has good photosynthetic ability and is known as the "Queen of Cereals" owing to its highest productivity. Maize is an important crop globally, it is the third most important cereal after wheat and rice in terms of area and production. It has got many industrial applications and is being used for extraction of starch, maltose syrup, glucose, oil etc. besides being used as human food and animal feed. The global statistics indicate the productivity of maize to be $5500 \mathrm{~kg} / \mathrm{ha}$, whereas India's productivity is $2510 \mathrm{~kg} / \mathrm{ha}$. In India, maize production is dominated by Karnataka, producing 15 per cent of India's maize while Tamil Nadu has the highest productivity of $6549 \mathrm{~kg} / \mathrm{ha}$. In Karnataka, maize occupies an area of 1.17 mha, with the production of $3.26 \mathrm{mt}$ and average productivity is $2773 \mathrm{~kg} / \mathrm{ha}$ (Anon., 2016).

Development of hybrid cultivars has accelerated the increase in productivity of maize across the world. Inbred lines are used for hybrid development and their worth is 
considered by their performance in combination with different other inbred lines. Combining ability of inbred lines gives information about genetic nature of quantitative traits and also conducive for selection of most appropriate parents to be used for heterosis breeding. The 'expected' value of any particular cross, according to Allard (1960), is the sum of the gca's of its two parental lines, while the deviation from this expected value is called sca. Predominance of gca is conducive for improvement of selection efficiency in segregating populations (Bocanski et al., 2009). Specific combining ability is cumulative performance of any two accessions in their specific hybrid combination. Combining ability is one of the powerful tools in identifying the best combiner that may be used in crosses either to exploitheterosis or accumulate fixable genes. Variance due to general combining ability (GCA) is indicator for extent of additive gene action while variance due to specific combing ability (SCA) shows the extent of nonadditive gene action. Additive and nonadditive types of gene actions are very important for genetic expression of yield and related traits. Selection of appropriate breeding program for maximum genetic improvement is based on relative values of general and specific combining abilities (Hayman, 1954; Griffing, 1956).

Line X Tester analysis, an extension of top cross method in which several testers are used (Kempthorne, 1957) to mate with selected inbred lines is very prominently used for estimation of combining ability of inbred lines and to make the selections easier. This design thus provides information about general and specific combining ability of parents and at the same time it is helpful in estimating various types of gene effects. It is very effective for identification of desired lines, so as to increase the frequency of targeted alleles in hybrids. Relative importance of additive and non-additive type of gene actions is also determined by this system of analysis. Being final product of complex physiological processes from sowing to maturity, grain yield has been reported to have a very complex mode of inheritance, thus different yield attributing traits were considered in this experiment for the evaluation of the parents for their combining abilities. Exploitation of hybrid vigour and selection of parents based on combining ability has been used as an important breeding approach in crop improvement. Breeder's objectives are to select hybrids on the basis of expected level of heterosis as well as specific combining ability. The present study involving a line $x$ tester analysis aimed to determine the general combining ability (gca) and specific combining ability (sca) of crosses for different traits.

\section{Materials and Methods}

Developing single cross hybrids requires promising inbred lines that have been tested for their gca effects, these when crossed with a diverse tester can be expected to give heterotic hybrids. But the main hurdle lies in the seed production of such single cross hybrids, as the in breds are generally low yielding and thus, making the hybrid seed production program expensive. Keeping in view this aspect, 16 promising CIMMYT and IIMR based germplasm lines were selected on the basis of their test weight and per se performance. These novel lines were crossed with 3 proven testers (CM-111, CM-500 \& PA-15) to produce 48 single cross experimental hybrids in Line $\times$ Tester design in rabi -2015. These 48 single cross hybrids, the 16 femalelines, 3 testers and 3 checks (GH-0727, CP-818 and NK-6240) were sown in Randomised Block Design in 3 replications across the three locations, viz., Agriculture Research Station, Arabhavi of Northern dry zone $\left(16^{\circ} 12^{\prime} \mathrm{N}\right.$ latitude and $74^{\circ} 57^{\prime} \mathrm{E}$ longitude, zone-3), Agriculture Research 
Station, Nippani of Northern Transition zone $\left(16^{\circ} 40^{\prime} \mathrm{N}\right.$ latitude and $74^{\circ} 38^{\prime} \mathrm{E}$ longitude, zone -8) and Main Agricultural Research Station (MARS), Dharwad of the Northern Transition zone $\left(15^{\circ} 26^{\prime} \mathrm{N}\right.$ latitude and $70^{\circ} 26^{\prime}$ E longitude, zone-8) of Karnataka during kharif-2016. Diversity prevailed with respect to the environment among the locations. Line $\times$ tester analysis, pooled over locations was run on Windostat, version 8.1 using the different yield and yield attributing traits that were observed during the crop growth and postharvest stages. The value of heterosis was calculated against the three commercial checks (standard heterosis). The details of the breeding materials used for the experimentation are mentioned in Table 1.

\section{Results and Discussion}

\section{Analysis of variance}

The analysis of variance for the 11 traits across environment revealed significant difference among the genotypes, indicating that there is significant variability among the genotypes under evaluation (Table 2). The environments were significantly different for all the characters except days to 50 per cent silking, shelling percentage and test weight. The environment $\mathrm{x}$ treatments was also significant for all characters except ear height and cob girth. This points out the differential expression of the genotypes across the three diverse locations chosen for this study. There was significant variation among the parents involved in crossing for all the characters except plant height, ear height, shelling percentage and 100 grain weight indicating greater diversity in the parental lines for the other traits. The mean sum of squares from parents' vs. crosses also indicated significant variations between the parents and crosses for all of the 11 traits that included days to $50 \%$ tasselling, days to $50 \%$ silking, plant height, ear height, cob length, cob girth, number of kernel rows per ear, number of kernels per ear and grain yield. This indicated their potential for exploitation through heterosis. The mean sum of squares of lines were highly significant for nine important traits like days to $50 \%$ tasselling, days to $50 \%$ silking, plant height, ear height, cob length, number of kernels per row, shelling percentage, 100 grain weight and grain yield which shows that lines chosen for this experiment were diverse from one another. The mean sum of squares for female vs. male parents was significant for the important yield and yield contributing traits viz., cob length, cob girth, number of kernel rows per ear, number of kernels per row, 100 grain weight. This shows that there is ample diversity among the lines and testers, thus one can expect crosses to be heterotic between them.

\section{Combining ability analysis}

Days from planting to flowering is one of the important maturity characters governing the earliness of a genotype. The traits like days to 50 per cent tasseling and days to 50per cent silking are desirable with significant combining ability in the negative direction. Line P-686 was significant across environments for days to 50 per cent tasselling, followed by P-768, P-679, P-750, P-778 and P-696. Tester CM-500 was also a significant combiner for this trait over pooled environment. For days to 50 per cent silking P-778 was the earliest followed by P-686, P750, P-679 and P-768. Among the testers, CM-500 was a promising tester for this trait. Line P-686 was the earliest line across environments. These lines were also showing earliness with respect to the checks (Table 3).

Plant height and ear height are desirable at the optimum, are directly related to yield and also helpful in mechanisation during harvest. But the optimum ear height with respect to the plant is the middle position or just above the middle of the plant. Even the significant negative combiners with shorter plant and ear 
height can be utilised as they show lodging resistance. The promising general combiners for plant height were P-596 and PA-15 (tester), for ear height P-596, P-683, P-693 and PA-15 were significant positive combiners. None of the parents were significantly good combiners at all the three locations for the traits of cob length and cob girth, lines; P-683 and P-668 were found to be promising combiners for location $\mathrm{E}_{1}$ and $\mathrm{E}_{2}$, similarly line P-750 was a significant combiner in $\mathrm{E}_{3}$ environment. These lines can be utilised for further improvement of the respective traits. None of the parents were significantly good combiners at all the three locations for the traits; cob length and cob girth. This can be explained by the difference in environment where the study has been undertaken. Important traits like number of kernel rows per cob and number of kernels per row exhibited very few good combiners. Only two lines; P-679 and P-684 were found to be good combiners for number of kernel rows per cob, whereas only line P-596 was good general combiner for the trait of number kernels per row.

Table.1 Materials used for the experimentation

\begin{tabular}{|c|c|}
\hline Lines & $\begin{array}{l}\text { P-596, P-683, P-668, P-679, P-684, P-687, P-693, P-694, P-696, } \\
\text { P-750, P-753, P-768, P-686, P-778, P-767, P-559 }\end{array}$ \\
\hline Testers & CM-111, PA-15, CM-500 \\
\hline Hybrids & $\begin{array}{l}\text { GH-1501, GH-1502, GH-1503, GH-1504, GH-1505, GH-1506, } \\
\text { GH-1507, GH-1508, GH-1509, } \\
\text { GH-1510, GH-1511, GH-1512, GH-1513, GH-1514, GH-1515, } \\
\text { GH-1516, GH-1517, GH-1518, } \\
\text { GH-1519, GH-1520, GH-1521, GH-1522, GH-1523, GH-1524, } \\
\text { GH-1525, GH-1526, GH-1527, } \\
\text { GH-1528, GH-1529, GH-1530, GH-1531, GH-1532, GH-1533, } \\
\text { GH-1534, GH-1535, GH-1536, } \\
\text { GH-1537, GH-1538, GH-1539, GH-1540, GH-1541, GH-1542, } \\
\text { GH-1543, GH-1544, GH-1545, } \\
\text { GH-1546, GH-1547, GH-1548 }\end{array}$ \\
\hline Checks & GH-0727, CP-818, NK-6240 \\
\hline
\end{tabular}


Table.2 Pooled analysis of variance for Line $\mathrm{x}$ Tester for different characters in maize

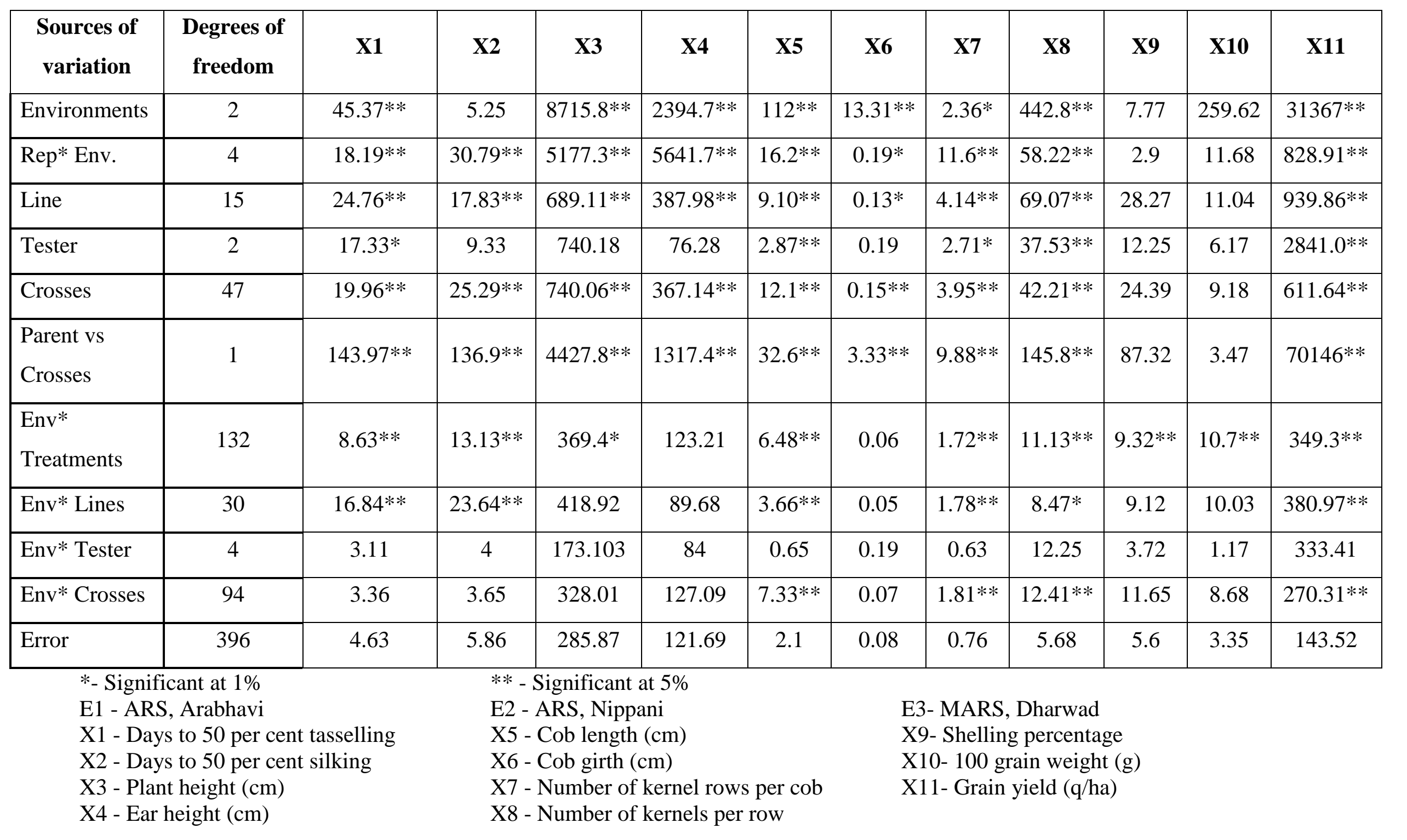


Table.3 Estimates of general combining ability (gca) effects of lines and testers for the 11 characters at different locations and Pooled over locations

\begin{tabular}{|c|c|c|c|c|c|c|c|c|c|c|c|}
\hline LINES & $\mathrm{X} 1$ & $\mathrm{X} 2$ & X3 & $\mathrm{X} 4$ & $\mathrm{X} 5$ & $\mathrm{X} 6$ & $\mathrm{X} 7$ & X8 & X9 & $\mathrm{X} 10$ & $\mathrm{X} 11$ \\
\hline $\mathrm{P}-559$ & -0.10 & -0.58 & $10.87 * *$ & $6.86 * *$ & 0.46 & -0.02 & -0.18 & $2.82 * *$ & $1.09 * *$ & -0.18 & 2.81 \\
\hline P-596 & $2.18 * *$ & $2.86 * *$ & $8.17 *$ & $5.60 * *$ & 0.52 & 0.06 & -0.08 & $1.13^{*}$ & -0.03 & -0.12 & $6.73 * *$ \\
\hline P-668 & 0.35 & 0.03 & 2.57 & 2.52 & 0.54 & -0.04 & -0.06 & $1.72 * *$ & -0.24 & 0.05 & 4.57 \\
\hline P-679 & $-0.93 * *$ & $-1.08 * *$ & -1.82 & -3.10 & $-0.66^{*}$ & 0.06 & $0.86 * *$ & $-2.07 * *$ & 0.19 & $1.71 * *$ & 4.58 \\
\hline P-683 & $0.73 *$ & $1.02 *$ & 4.15 & 1.78 & $1.80 * *$ & -0.00 & -0.15 & 0.82 & 0.07 & -0.79 & $5.98 *$ \\
\hline P-684 & $1.40 * *$ & $1.25 * *$ & 4.39 & 2.84 & 0.42 & $0.11^{*}$ & $0.73 * *$ & 0.87 & -0.63 & $0.93 *$ & 4.08 \\
\hline P-686 & $2.01 * *$ & $2.47 * *$ & 5.93 & $6.26 * *$ & -0.20 & $-0.12 *$ & 0.19 & 0.39 & -0.24 & 0.16 & 0.93 \\
\hline P-687 & $1.18 * *$ & $1.02 *$ & 6.00 & 2.32 & -0.15 & -0.02 & -0.15 & 0.01 & -0.30 & -0.10 & 0.02 \\
\hline P-693 & $-0.81^{*}$ & -0.42 & -1.63 & 2.96 & $-0.80 * *$ & -0.09 & -0.20 & $-1.31 * *$ & 0.29 & 0.05 & $7.84 * *$ \\
\hline P-694 & $-0.93 * *$ & $-1.52 * *$ & $-6.56^{*}$ & -2.42 & $-0.61 *$ & $0.18^{*}$ & $-0.46 * *$ & -0.50 & 0.66 & $-1.17 *$ & 2.74 \\
\hline P-696 & 0.35 & 0.36 & 2.03 & 1.54 & -0.04 & -0.03 & 0.16 & 0.87 & 0.23 & $-1.06^{*}$ & 4.14 \\
\hline P-750 & $-1.37 * *$ & $-1.19 * *$ & -4.81 & $-5.04 *$ & -0.2 & -0.04 & -0.26 & $-1.79 * *$ & -0.60 & 0.33 & 2.98 \\
\hline P-753 & $-2.26 * *$ & $-1.75^{* *}$ & -4.28 & $-4.51 *$ & -0.34 & -0.05 & -0.27 & $-1.04^{*}$ & $-1.00 * *$ & 0.16 & $6.06^{*}$ \\
\hline $\mathrm{P}-767$ & $-0.93 * *$ & $-1.86 * *$ & $-9.27 * *$ & $-6.35 * *$ & 0.04 & -0.01 & $-0.71 * *$ & -0.41 & $1.03 * *$ & $1.88 * *$ & $5.87 *$ \\
\hline P-768 & -0.65 & -0.08 & $-12.2 * *$ & $-7.87 * *$ & 0.10 & 0.05 & 0.21 & -0.66 & 0.31 & -0.12 & 0.32 \\
\hline P-778 & -0.21 & -0.53 & -3.54 & -3.40 & $-0.88^{* *}$ & -0.03 & $0.37 *$ & -0.85 & $-0.84 *$ & $-1.73 * *$ & 2.34 \\
\hline \multicolumn{12}{|l|}{ TESTERS } \\
\hline T-CM111 & -0.27 & -0.15 & -1.74 & -0.89 & $0.78 * *$ & -0.04 & $-0.17 *$ & -0.10 & -0.09 & 0.00 & $-3.92 * *$ \\
\hline T-PA15 & $0.59 * *$ & $0.62 * *$ & $4.90 * *$ & $3.06 * *$ & $-0.25^{*}$ & 0.03 & $0.22 * *$ & 0.09 & 0.23 & 0.18 & $7.18^{* *}$ \\
\hline T-CM500 & $-0.33 *$ & $-0.47 * *$ & $-3.16^{*}$ & $-2.17 *$ & $-0.53 * *$ & 0.02 & -0.05 & 0.01 & -0.14 & -0.18 & $-3.26 * *$ \\
\hline CD at $5 \%$ female & 0.69 & 0.81 & 6.30 & 4.20 & 0.58 & 0.11 & 0.33 & 0.89 & 0.67 & 0.89 & 4.96 \\
\hline CD at $1 \%$ female & 0.91 & 1.07 & 8.30 & 5.54 & 0.77 & 0.15 & 0.44 & 1.17 & 0.88 & 1.18 & 6.54 \\
\hline S.Em \pm & 0.35 & 0.41 & 3.20 & 2.13 & 0.30 & 0.06 & 0.17 & 0.45 & 0.34 & 0.45 & 2.52 \\
\hline $\mathrm{CD}$ at $5 \%$ male & 0.30 & 0.35 & 2.73 & 1.81 & 0.25 & 0.05 & 0.14 & 0.39 & 0.29 & 0.39 & 2.15 \\
\hline $\mathrm{CD}$ at $1 \%$ male & 0.39 & 0.46 & 3.59 & 2.40 & 0.33 & 0.06 & 0.19 & 0.51 & 0.38 & 0.51 & 2.83 \\
\hline S.Em \pm & 0.15 & 0.18 & 1.39 & 0.92 & 0.13 & 0.03 & 0.07 & 0.20 & 0.15 & 0.20 & 1.09 \\
\hline
\end{tabular}




\section{Int.J.Curr.Microbiol.App.Sci (2017) 6(10): 2608-2618}

Table.4 Estimates of specific combining ability (sca) effects for 11 traits at individual environments and pooled over environments

\begin{tabular}{|c|c|c|c|c|c|c|c|c|c|c|c|}
\hline Hybrids & $\mathrm{X} 1$ & $\mathrm{X} 2$ & X3 & $\mathrm{X} 4$ & $\begin{array}{l}\mathrm{X} 5 \\
\end{array}$ & X6 & $\begin{array}{l}\mathrm{X} 7 \\
\end{array}$ & $\mathrm{X} 8$ & $\begin{array}{l}\mathrm{X} 9 \\
\end{array}$ & $\begin{array}{l}\mathrm{X} 10 \\
\end{array}$ & $\mathrm{X} 11$ \\
\hline GH-1501 & 0.04 & -0.35 & 0.965 & 0.501 & -0.066 & 0.007 & -0.557 & 0.6 & -0.549 & 1.11 & -0.37 \\
\hline GH-1502 & -0.31 & -0.29 & -0.205 & 0.211 & 0.559 & -0.001 & 0.234 & $\begin{array}{l}-0.594 \\
\end{array}$ & -0.434 & -0.57 & 2.02 \\
\hline GH-1503 & 0.27 & 0.64 & -0.759 & -0.711 & -0.493 & -0.006 & 0.323 & -0.006 & 0.984 & -0.55 & -1.65 \\
\hline GH-1504 & 0.26 & 0.36 & 5.668 & 4.696 & -0.639 & -0.059 & 0.243 & $-3.217 * *$ & -0.843 & $2.223 * *$ & 3.61 \\
\hline GH-1505 & $1.23^{*}$ & 1.26 & 2.091 & 0.074 & -0.444 & -0.033 & -0.199 & -0.01 & 0.152 & -1.29 & -3.79 \\
\hline GH-1506 & $-1.50^{*}$ & $-1.6^{*}$ & $\begin{array}{l}-7.759 \\
\end{array}$ & -4.769 & $1.083 *$ & 0.093 & -0.044 & $3.227^{* *}$ & 0.691 & -0.94 & 0.19 \\
\hline GH-1507 & 0.26 & 0.53 & -6.613 & -4.984 & -0.973 & -0.041 & -0.279 & -1.4 & 0.799 & -1.44 & -5.37 \\
\hline GH-1508 & -0.09 & -0.40 & 5.58 & 5.268 & $1.158 *$ & 0.13 & $1.045 * *$ & -0.144 & 1.018 & 0.21 & -0.09 \\
\hline GH-1509 & -0.17 & -0.13 & 1.033 & -0.284 & -0.186 & -0.089 & $-0.766 * *$ & $1.544 *$ & $-1.817 * *$ & 1.23 & 5.46 \\
\hline GH-1510 & $1.37^{*}$ & 0.47 & 2.806 & 0.495 & -0.449 & 0.12 & -0.168 & -0.45 & 0.076 & 1.06 & $8.765 *$ \\
\hline GH-1511 & -0.65 & -0.12 & -0.593 & -0.844 & 0.266 & -0.088 & -0.044 & 0.456 & -0.471 & -1.45 & $\begin{array}{l}-3.78 \\
\end{array}$ \\
\hline GH-1512 & -0.72 & -0.35 & -2.213 & 0.349 & 0.183 & -0.032 & 0.211 & -0.006 & 0.395 & 0.40 & -4.99 \\
\hline GH-1513 & -0.62 & -0.96 & -5.122 & -5.672 & $1.745 * *$ & -0.006 & -0.34 & $-1.650 *$ & -0.453 & 0.56 & -8.42 \\
\hline GH-1514 & 0.01 & -0.73 & 2.708 & 2.41 & -0.986 & 0.165 & $1.184 * *$ & 1.406 & 0.036 & -1.12 & $10.088 *$ \\
\hline GH-1515 & 0.60 & $1.70^{*}$ & 2.414 & 3.262 & $\begin{array}{l}-0.76 \\
\end{array}$ & $\begin{array}{l}-0.159 \\
\end{array}$ & $-0.844 * *$ & 0.244 & 0.417 & 0.56 & -1.67 \\
\hline GH-1516 & -0.12 & -0.18 & 5.681 & 1.252 & -0.096 & 0.115 & -0.451 & 1.3 & -0.019 & $2.168 * *$ & 5.35 \\
\hline GH-1517 & 1.18 & 1.37 & 1.992 & 3.875 & 0.09 & 0.022 & 0.406 & -1.344 & 0.186 & 1.16 & 1.41 \\
\hline GH-1518 & -1.05 & -1.18 & $\begin{array}{l}-7.673 \\
\end{array}$ & -5.126 & 0.006 & $\begin{array}{l}-0.137 \\
\end{array}$ & 0.045 & $\begin{array}{c}0.044 \\
\end{array}$ & $\begin{array}{l}-0.166 \\
\end{array}$ & $-3.325 * *$ & -6.76 \\
\hline GH-1519 & 0.60 & 0.09 & 3.431 & 5.665 & $1.794 * *$ & $0.208 *$ & 0.393 & $5.233 * *$ & $1.824 * *$ & 1.11 & $13.049 * *$ \\
\hline GH-1520 & -0.09 & 0.15 & 5.632 & -0.45 & -0.935 & -0.064 & -0.299 & $-2.010 *$ & -0.459 & $-1.565 *$ & -5.52 \\
\hline GH-1521 & -0.50 & -0.24 & -9.063 & -5.215 & -0.858 & -0.144 & $\begin{array}{c}-0.094 \\
\end{array}$ & $-3.223 * *$ & $-1.365 *$ & 0.45 & -7.53 \\
\hline GH-1522 & $\begin{array}{c}-0.06 \\
\end{array}$ & 0.70 & $\begin{array}{l}-1.532 \\
\end{array}$ & $\begin{array}{l}-3.107 \\
\end{array}$ & \begin{tabular}{|c|}
-0.761 \\
\end{tabular} & 0.027 & 0.766 ** & $-2.533 * *$ & 1.069 & -0.29 & -3.56 \\
\hline GH-1523 & 0.06 & -0.23 & -7.294 & -2.105 & -0.505 & 0.009 & -0.394 & $-1.727 *$ & -0.332 & -0.14 & 3.96 \\
\hline GH-1524 & -0.01 & -0.46 & 8.826 & 5.212 & $1.266 *$ & -0.036 & -0.372 & $4.260 * *$ & -0.737 & 0.43 & -0.40 \\
\hline GH-1525 & 0.76 & 0.47 & 7.397 & 4.326 & 0.241 & 0.049 & -0.057 & 0.533 & 0.293 & -0.78 & 2.15 \\
\hline GH-1526 & -0.59 & -0.62 & $-12.107 *$ & $-8.992 *$ & 0.476 & $\begin{array}{l}-0.095 \\
\end{array}$ & $-0.699 *$ & $\begin{array}{l}0.99 \\
\end{array}$ & $\begin{array}{l}-0.464 \\
\end{array}$ & 0.55 & $\begin{array}{l}-2.40 \\
\end{array}$ \\
\hline GH-1527 & -0.17 & 0.14 & 4.71 & 4.666 & -0.717 & 0.046 & $0.756^{* *}$ & -1.523 & 0.171 & 0.23 & 0.25 \\
\hline GH-1528 & -0.95 & -0.57 & -6.221 & -0.513 & -0.294 & $-0.284 * *$ & 0.577 * & 0.567 & -0.423 & -1.06 & -3.90 \\
\hline GH-1529 & -0.15 & -1.18 & -1.205 & -3.045 & $1.126 *$ & $\begin{array}{l}-0.028 \\
\end{array}$ & 0.134 & $1.873 *$ & -0.164 & $3.435 * *$ & -4.50 \\
\hline GH-1530 & 1.10 & $1.75^{*}$ & 7.426 & 3.558 & -0.832 & $0.312 * *$ & $-0.711 *$ & $-2.440 * *$ & 0.587 & $-2.381 * *$ & 8.40 \\
\hline GH-1531 & 0.26 & $1.53^{*}$ & -6.11 & -5.893 & 0.219 & 0.03 & 0.138 & 0.3 & -0.558 & -1.33 & -4.55 \\
\hline GH-1532 & $-1.43^{*}$ & -0.40 & 4.128 & 3.285 & 0.085 & 0.032 & -0.305 & 0.856 & 0.287 & -0.34 & 3.66 \\
\hline GH-1533 & 1.16 & -1.13 & 1.982 & 2.607 & -0.304 & -0.062 & 0.167 & -1.156 & 0.272 & $1.675 *$ & 0.90 \\
\hline GH-1534 & -0.01 & -0.9 & 2.026 & 0.704 & 0.733 & $\begin{array}{ll}0.017 \\
\end{array}$ & 0.355 & 1.117 & -1.569 ** & 0.45 & 0.39 \\
\hline GH-1535 & -0.04 & 0.65 & -1.514 & 2.488 & $-1.227 *$ & -0.025 & $-0.772 * *$ & $-2.027 *$ & 0.332 & $-1.898 *$ & -2.42 \\
\hline GH-1536 & 0.05 & 0.25 & -0.512 & -3.191 & 0.494 & 0.008 & 0.417 & 0.91 & $1.238 *$ & 1.45 & 2.02 \\
\hline GH-1537 & 0.54 & 0.31 & 7.718 & 6.063 & -0.977 & 0.005 & $0.766 * *$ & -1.083 & $-1.312 *$ & -0.22 & -4.51 \\
\hline GH-1538 & -0.65 & -1.29 & -10.526 & -7.19 & $1.285 *$ & 0.032 & $-0.594 *$ & 1.223 & 0.496 & $1.602 *$ & 1.29 \\
\hline GH-1539 & 0.10 & 0.97 & 2.809 & 1.126 & -0.308 & -0.037 & -0.172 & -0.14 & 0.816 & -1.38 & 3.22 \\
\hline GH-1540 & 0.05 & -0.41 & -8.552 & -5.988 & 0.021 & -0.046 & 0.032 & 0.783 & -0.609 & -0.28 & 1.50 \\
\hline GH-1541 & -0.48 & 0.65 & 8.271 & \begin{tabular}{|l|l|}
5.259 \\
\end{tabular} & -0.079 & $\begin{array}{l}-0.06 \\
\end{array}$ & -0.06 & 0.34 & 0.255 & -0.62 & -0.80 \\
\hline GH-1542 & 0.44 & -0.24 & 0.28 & 0.729 & 0.058 & 0.106 & 0.028 & -1.123 & 0.355 & 0.90 & -0.70 \\
\hline GH-1543 & -0.73 & -0.02 & -2.853 & 0.429 & -0.681 & -0.128 & $-0.895 * *$ & $-1.667 *$ & 0.566 & $-1.777 *$ & -2.63 \\
\hline GH-1544 & 0.90 & 0.54 & -0.652 & -2.479 & 0.24 & -0.012 & 0.278 & $1.890 *$ & 0.158 & $1.713 *$ & 3.58 \\
\hline GH-1545 & -0.17 & -0.52 & 3.505 & 2.05 & 0.442 & 0.14 & $0.617 *$ & -0.223 & -0.723 & 0.06 & -0.95 \\
\hline GH-1546 & $-1.67 * *$ & -1.07 & 1.31 & 2.026 & 0.183 & -0.015 & -0.523 & $1.567 *$ & $1.710^{* *}$ & -1.50 & -1.50 \\
\hline GH-1547 & 1.12 & 0.65 & 3.696 & 2.236 & $-1.107 *$ & 0.017 & 0.084 & -1.177 & -0.594 & 0.32 & -2.72 \\
\hline $\begin{array}{l}\text { GH-1548 } \\
\end{array}$ & 0.55 & 0.42 & -5.006 & -4.262 & 0.924 & -0.003 & 0.439 & -0.39 & -1.117 & $\begin{array}{l}1.18 \\
\end{array}$ & 4.22 \\
\hline C.D. at $1 \%$ & 1.57 & 1.85 & 14.3715 & 9.5884 & 1.3288 & 0.259 & 0.7541 & 2.0329 & 1.5194 & 2.0354 & 11.3287 \\
\hline C.D. at 5\% & 1.19 & 1.40 & 10.908 & 7.278 & 1.009 & 0.197 & 0.572 & 1.543 & 1.153 & 1.545 & 8.599 \\
\hline S.Em. \pm & 0.60 & 0.71 & 5.5417 & 3.6973 & 0.5124 & 0.099 & 0.2908 & 0.7839 & 0.5859 & 0.7849 & 4.3684 \\
\hline
\end{tabular}


Table.5 Estimates of variance components as reference to the prevailing gene action

\begin{tabular}{|l|c|c|c|}
\hline & $\sigma_{\text {gca }}^{2}$ & $\sigma_{\text {sca }}^{2}$ & $\sigma_{\text {gca/ }}^{2} \sigma_{\text {sca }}^{2}$ \\
\hline Days to 50 per cent tasseling & 0.43 & 0.44 & 0.98 \\
\hline Days to 50 per cent silking & 0.53 & 0.51 & 1.04 \\
\hline Plant height (cm) & 19.16 & 14.14 & 1.35 \\
\hline Ear height (cm) & 8.29 & 9.52 & 0.87 \\
\hline Cob length (cm) & 0.45 & 0.65 & 0.68 \\
\hline Cob girth (cm) & 0.01 & 0.01 & 0.19 \\
\hline Number of kernel rows per cob & 0.05 & 0.30 & 0.17 \\
\hline Number of kernels per row & 0.22 & 4.07 & 0.05 \\
\hline Shelling percentage & 0.05 & 0.64 & 0.09 \\
\hline 100 seed weight (g) & 0.10 & 2.22 & 0.05 \\
\hline Grain yield per hectare (q) & 34.03 & 13.97 & 1.05 \\
\hline
\end{tabular}

Table.6 Top ten heterotic hybrids for grain yield across locations and pooled over locations

\begin{tabular}{|c|c|c|c|c|c|}
\hline Hybrids & & & \multicolumn{3}{|c|}{ Standard heterosis } \\
\hline & $\begin{array}{c}\text { Grain yield } \\
(\mathbf{q} / \mathbf{h a})\end{array}$ & $\mathbf{s c a}$ & $\begin{array}{c}\text { GH- } \\
\mathbf{0 7 2 7}\end{array}$ & $\mathbf{C P - 8 1 8}$ & NK-6240 \\
\hline GH-1514 & 91.32 & $10.08^{*}$ & $53.77^{* *}$ & $26.57 * *$ & 10.53 \\
\hline GH-1532 & 83.02 & 3.66 & $39.83^{* *}$ & 15.1 & 0.51 \\
\hline GH-1517 & 80.74 & 1.41 & $35.95^{* *}$ & 11.91 & -2.28 \\
\hline GH-1502 & 80.08 & 2.02 & $34.84^{* *}$ & 10.99 & -3.08 \\
\hline GH-1508 & 79.73 & -0.09 & $34.26^{* *}$ & 10.51 & -3.5 \\
\hline GH-1523 & 79.22 & 3.96 & $33.41^{* *}$ & 9.81 & -4.11 \\
\hline GH-1544 & 79.15 & 3.58 & $33.29^{* *}$ & 9.71 & -4.2 \\
\hline GH-1505 & 78.18 & -3.79 & $31.66^{* *}$ & 8.37 & -5.37 \\
\hline GH-1519 & 76.27 & $13.04 * *$ & $28.43^{* *}$ & 5.71 & -7.69 \\
\hline GH-1547 & 74.9 & -2.72 & $26.08^{*}$ & 3.78 & -9.38 \\
\hline
\end{tabular}


Fig.1 Level of heterosis and sca effects of top ten promising hybrids

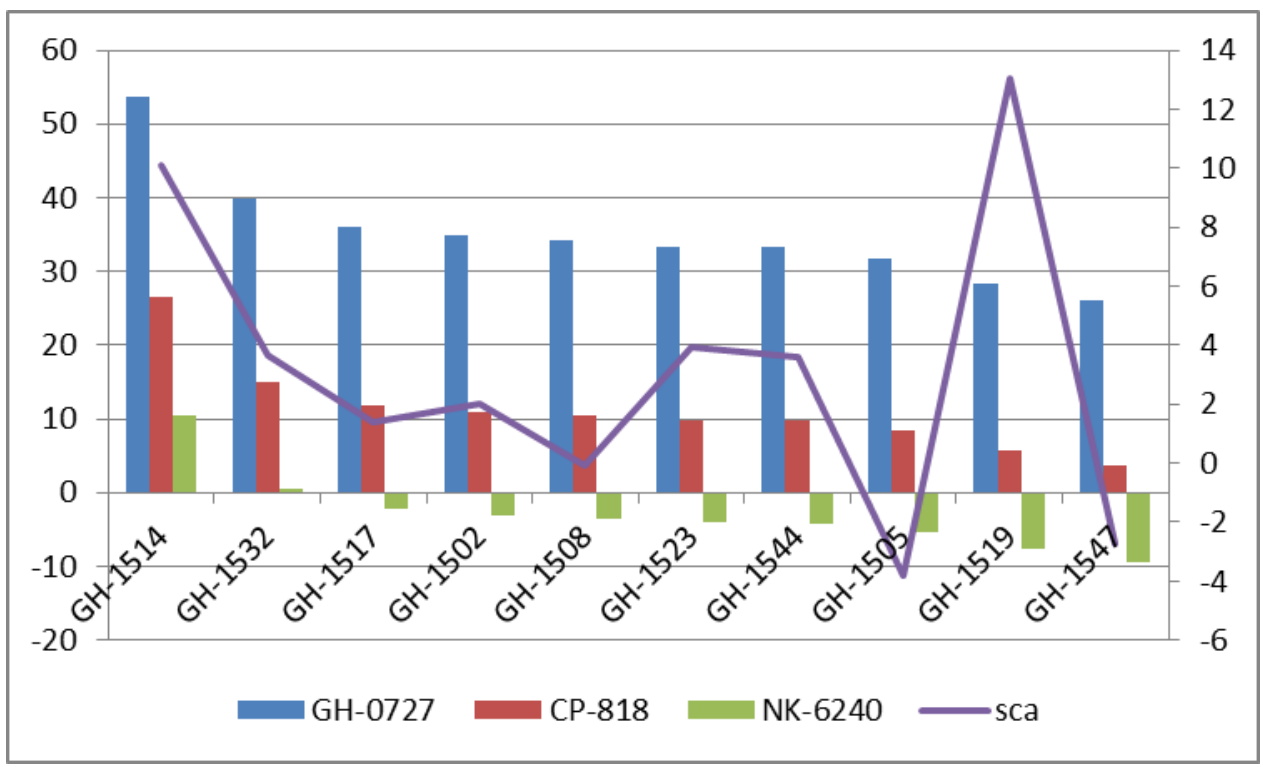

The trait 100 grain weight exhibited a few good general combiners, out of which the lines P-679 and P-778 were the best combiner, also had the high mean weight for the trait. This line can be utilised for the improvement of the trait. Grain yield trait exhibited different good general combiners among the three locations. The pooled analysis showed P-679 to be a good combiner for the trait, this line was also a good combiner for 100 grain weight. It can be inferred that this line has the potential as a good combiner for improving yield. Such high yielding in breds can be useful to mitigate the problem of seed yield for single cross hybrids. Tester PA-15 was very promising as the top high yielding hybrids (GH-1502, GH-1512, GH-1514 and GH1532) were produced by crossing various lines with this tester. This tester may be considered to be diverse enough from the female inbred lines so as to produce high yielding crosses as compared to the checks.

To understand the relationship seemed between per se performance and sca effects, crosses under pooled environment were taken into account. Specific combining ability effects for traits like earliness were significant for very few crosses, this may be due to the low narrow sense heritability of the trait as reported earlier by workers like Mohammad and Ismail (2014). GH-1506 showed significant values in the negative direction contributing to its earliness. For both the traits; plant height and ear height hybrid $\mathrm{GH}-$ 1524 was significant in $\mathrm{E}_{1}$ environment. The other environments did not exhibit any significant crosses for these traits. Crosses GH-1513 and GH-1519 exhibited significant sca effects for cob length, crosses GH-1519 and $\mathrm{GH}-1530$ exhibited significant sca effects for cob girth. The hybrids of these crosses also displayed higher per se mean for the respective characters. The crosses showing significant specific sca effects were GH-1508, GH-1514, GH-1522, GH-152, GH-1537 for number of kernel rows per ear. Similarly for number kernels per row, the crosses that can be termed as good specific combiners were GH-1506, GH-1509, GH-1519 and GH-1524. For yield the best specific combiners were GH-1514, GH-1519 andGH-1510, as well as with highper se mean for the trait. 
The analysis of GCA, SCA variances and their ratios revealed the type of gene actions involved in the expression of various traits. The SCA variance was higher than GCA variance for traits like ear height, cob length, number of kernel rows per ear, number kernels per row, shelling percentage, 100 grain weight. This shows the preponderance of non-additive gene action for these traits. For other characters like days to $50 \%$ silking and plant height the pooled ratio was more than unity showing underlying additive gene action for this trait. The results also revealed that GCA variance was higher than SCA variance and GCA/SCA ratio was more than unity for grain yield. This would indicate that the additive gene action played the major role than non-additive gene action in governing the inheritance of these traits. The closer the ratio of GCA:SCA is to unity, the greater the predictability of progeny performance based on the GCA alone and the better the transmission of trait to the progenies. Less than one ratio of GCA: SCA observed in all environments for all characters revealed that that selection for these characters should be performed using recurrent selection method. This result supports the finding of Geetha (1997) and El-Badawy (2012) for grain yield (Table 4).

\section{Heterosis}

Presence of significant differences among parents and crosses revealed the choice of exploitation of heterosis for all the characters studied. Heterosis in a positive direction is desirable for most of the traits, however heterosis in negative direction is desirable for traits like earliness. The top ten heterotic hybrids (standard heterosis over the three checks) with respect to their grain yield and the respective sca effects of each of the crosses are given in Table 5. Traits like yield, 100 grain weight, number of kernel rows and number of kernels per row are more important for a hybrid development point of view. Yield was higher in case of the hybrid GH-1514 across location over the best check NK-6240, followed by GH-1532 and GH-1535 (Table $6)$.

At least part of the genes associated with this trait have dominance gene action, and the inbred lines involved in this cross are genetically divergent among themselves, which was expected, since they were selected from diverse groups. Then, the significant sca value of this cross along with positive and high gca values from its inbred lines, significant gca value of the tester afforded this single-cross to be the highest yielding in this set. Thus, both additive and non-additive effects (dominance and epistasis) contributed for the high productivity of this single-cross, which was also more productive than the superior commercial hybrid, NK-6240. The same trend was seen in case of number of kernel rows per cob, GH-1514 proved to have higher heterosis with a value of $10.08 \%$ over the best check (NK-6240) for yield.

The results from pooled analysis have highlighted lines; P-679 as a significant positive combiner for 100 grain weight and also with high per se performance. This line can be useful for improving the trait through recurrent selection. Hybrid GH-1514 with significant positive sca effect was the highest yielding heterotic cross as compared to the checks, thus this hybrid can be advanced for future breeding programs.

\section{References}

Allard, R. W., and Bradshaw, A. D., 1964. Implications of genotype environment interaction in applied plant breeding. Crop Sci., 4: 503-508.

Aminu, D., and Izge, A. U., 2013. Gene action and heterosis for yield and yield traits in maize (Zea mays L.) under 
drought conditions in Northern Guinea and Sudan Savannas of Borno State, Nigeria Peak J. Agric. Sci., 1 (1): 17-23. Anon., 2016. Annu.Prog. Rep. Maize, IIMR, Ludhiana.

Bocanski, J., Z. Sreckov, and A. Nastasic 2009.Genetic and phenotypic relationship between grain yield and components of grain yield of maize (Zea mays L.). Genetika41 (2): 145-154.

El-Badawy, 2013. Heterosis and combining ability in maize using diallel crosses among seven new inbred Lines. Asian $J$. Crop Sci., 5 (1): 1-13.

Geetha, K., 1997. Genetic analysis of yield, starch and downy mildew resistance in maize (Zea mays L.).Ph. D. Thesis, Tamil Nadu Agric. Univ., Coimbatore.

Griffing, B., 1956. Concept of general and specific combining ability in relation to diallel crossing system. Aust. J. Biol. Sci. 9:463-493.

Hayman, B.I., 1954. The theory and analysis of diallelcrosses. Genetics, 39: 789-809.

Kambe, G. R., Kage, U., Lohithaswa, H. C., Shekara, B. G. and Shobha, D., 2013. Combining ability studies in maize (Zea mays L.). Mol. Pl. Breed. 3 (14):116127.

Kempthorne, O., 1957. An introduction to genetic statistics. New York, John Wiley and Sons, Inc. London: Chapman and Hall Limited.

Mohammed, A. H., and Ismail, H. A., 2014. Combining ability, gene action and heterosis in some inbred lines of maize at two sowing dates using factorial mating design. Int. J. Pure Appl. Sci. Technol., 21(1): 17-30.

\section{How to cite this article:}

Siddharth Panda, Mruthunjaya C. Wali, R.M. Kachapur and Harlapur, S.I. 2017. Combining Ability and Heterosis Analysis of Single Cross Hybrids of Maize (Zea mays L.). Int.J.Curr.Microbiol.App.Sci. 6(10): 2608-2618. doi: https://doi.org/10.20546/ijcmas.2017.610.307 